Service social

\title{
Les réfugiés au Québec, du discours à la réalité
}

\section{André Jabob}

Volume 40, numéro 3, 1991

Oppression, intolérance et intervention

URI : https://id.erudit.org/iderudit/706545ar

DOI : https://doi.org/10.7202/706545ar

Aller au sommaire du numéro

\section{Éditeur(s)}

École de service social de l'Université Laval

ISSN

1708-1734 (numérique)

Découvrir la revue

Citer cet article

Jabob, A. (1991). Les réfugiés au Québec, du discours à la réalité. Service social, 40(3), 68-81. https://doi.org/10.7202/706545ar

\section{Résumé de l'article}

L'auteur traite de la question des personnes réfugiées, en confrontant le discours et la réalité. Ici, le discours officiel n'est pas ouvertement attestataire d'intolérance. Il est ambivalent et même plutôt humanitaire. La réalité, elle, est oppressive, notamment à travers le caractère sélectif des procédures, leur longueur et leur lourdeur bureaucratique, leur tendance discriminatoire une fois les personnes entrées au pays. En ce qui concerne les services sociaux spécialisés à leur disposition, l'auteur constate qu'ils relèvent pour la plupart d'organismes non gouvernementaux, obligés de composer avec un sous-financement chronique. Pour continuer à être financés, ils doivent " prouver leur efficacité en offrant des services dans des conditions qui seraient jugées inacceptables par des professionnels et des employés du gouvernement ». Ils doivent aussi pour les mêmes raisons " se plier à une forme de soumission idéologique et politique ». L'auteur identifie ici le lieu d'un partenariat à redéfinir.
Ce document est protégé par la loi sur le droit d'auteur. L'utilisation des services d'Érudit (y compris la reproduction) est assujettie à sa politique d'utilisation que vous pouvez consulter en ligne.

https://apropos.erudit.org/fr/usagers/politique-dutilisation/ 
André Jacob, professeur au Département de travail social, Université du Québec à Montréal.

\section{Les réfugiés au Québec, du discours à la réalité}

André Jacob

Les réfugiés ont toujours formé une portion significative des contingents d'immigrants au Canada depuis la dernière guerre mondiale. Les années qui ont suivi la Deuxième Guerre mondiale ont vu défiler des millions de "déplacés " européens à travers le monde, particulièrement en Amérique du Nord, en Amérique du Sud et en Australie. Ces Européens ont contribué à enrichir les Amériques par leurs avoirs et leur savoir-faire. Cette armée de travailleurs et $d^{\prime}$ investisseurs correspondait au profil espéré d'un futur citoyen de l'Amérique : origine européenne, expérience de travail ou capacité d'investissement et, dans plusieurs cas, anti-communisme pour ceux qui fuyaient l'Europe de l'Est; cette dernière caractéristique s'inscrivait dans le cadre idéologique de la reconstruction de l'Europe, le développement des deux grands blocs militaro-politiques Est-Ouest durant la guerre froide.

Au Canada, à partir de 1973, la situation change radicalement avec l'arrivée des premiers réfugiés fuyant le Chili du général Pinochet. Ces quelques milliers de réfugiés présentent d'autres caractéristiques: visibles par la couleur de leur peau ou leurs traits (Africains, Asiatiques et Latino-Américains), différents par leurs expériences de vie, professionnelles et politiques (bon nombre fuient des régimes répressifs soutenus par les pays du Nord). En général, l'opinion publique semble favorable à l'acceptation de ces premiers réfugiés venus du Sud et les gouvernements répondent de façon positive. En 1975, la fin de la guerre du Viet-Nam annonce l'entrée d'une nouvelle vague de réfugiés, les « boat-people » vietnamiens suivis de Cambodgiens (1979), visibles et originaires d'une société extrêmement différente du contexte dominant nord-américain sur le plan social, culturel, économique et politique. L'arrivée de tels groupes illustre le « choc culturel » 
que vient créer leur insertion sociale dans les grandes villes. Le phénomène allait susciter de nouvelles attitudes dans la population, notamment une grande sympathie, et des politiques et structures d'accueil nouvelles allaient voir le jour. Depuis, des dizaines de milliers de réfugiés "visibles » de divers pays du tiers monde (Éthiopie, Somalie, Ghana, Haïti, El Salvador, Guatemala, Cambodge, Laos, Chine, Iran, etc.) grossissent les rangs des minorités visibles. En moyenne, environ 10000 réfugiés par an entrent au Canada.

On observe le même phénomène en Europe. Le Haut Conseil à I'intégration (H.C.I.) souligne le phénomène dans son dernier énoncé de politique :

L'augmentation des demandes d'asile s'observe dans tous les pays d'Europe du Nord, et ce, quelles que soient les législations en vigueur. L'Allemagne a accueilli plus de 100000 demandeurs d'asile politique en 1988, plus de 120000 en 1989 et 190000 en 1990. (H.C.I., $1991: 35)$

En ce qui concerne la France, on a enregistré 34000 demandes d'asile en 1988 et 61000 en 1989 (H.C.I., 1991 : 34). En outre, il est intéressant d'observer que la France a accordé le statut de réfugiés à seulement 8714 personnes en 1989, chiffre à peu près équivalent à celui des années précédentes; en somme, $72 \%$ des demandes ont été rejetées. Aux États-Unis, la situation s'était améliorée au début des années 80, car la porte était alors grande ouverte pour les ressortissants des pays d'Europe de l'Est, tout en demeurant cependant étroitement fermée pour les Latino-Américains; par exemple, en 1981, seulement 2 Salvadoriens sur 510 ont été acceptés et en 1983, 328 sur 13045 et 23 Haïtiens sur 375 . Aujourd'hui, les changements survenus à l'Est ont entraîné un durcissement de la politique de contrôle des frontières. Seuls les immigrants investisseurs et les personnes dûment qualifiées sont les bienvenus. Cette situation ne souffre aucune comparaison avec ce qui a cours au Canada où à peine $10 \%$ des demandes étaient refusées jusqu'à la fin de 1990. Depuis le début de 1991, on observe cependant un contrôle plus sévère et une augmentation graduelle du nombre de refus.

L'acceptation de quelques milliers de demandeurs d'asile ne signifie pas pour autant que le Canada et le Québec font preuve d'une générosité débordante; un regard critique sur les données actuellement disponibles montrent que le Canada et le Québec, tout comme les États-Unis et les pays développés de l'Europe de l'Ouest, ne sont pas particulièrement accueillants. Quoi qu'il en soit, le mouvement des réfugiés dans le monde s'accentue et engendre de nouveaux problèmes sociaux; d'où la nécessité pour les gouvernements et les diverses institutions de services d'analyser les situations sociales vé- 
cues par ces gens et de mettre en place de nouvelles politiques et des programmes d'intervention adaptés.

Dans cet article, je tenterai donc de dégager quelques contradictions entre le discours officiel et les pratiques à l'égard des réfugiés. Dans un premier temps, je définirai quelques pistes d'analyse de la problématique de l'insertion sociale des réfugiés. En second lieu, je montrerai les contradictions qui traversent les pratiques sociales et économiques à l'égard des réfugiés.

\section{Les réfugiés, des itinérants sur la planète}

Au cours des cinq dernières années, le nombre de réfugiés dans le monde a doublé, passant de 7 millions et demi à 15 millions qui sont techniquement considérés comme réfugiés, c'est-à-dire plus ou moins recensés. En outre, le Haut-Commissariat pour les réfugiés des $\mathrm{Na}$ tions-Unies estime qu'autant de gens vivent dans des conditions semblables à celles de réfugiés à l'étranger (CCR, juin $1990: 4)$. Des millions de personnes errent d'un pays à l'autre, sans passeport, sans aucune protection légale ou sociale.

Le Conseil canadien pour les réfugiés (CCR) estime qu'un " grand nombre de personnes d'Amérique centrale, par exemple, n'ont pas demandé l'asile dans aucun pays, à cause des procédures inadéquates en matière de détermination du statut de réfugié ou du faible taux d'approbation du statut de réfugié dans les pays où ils vont. Leur nombre se situe quelque part entre 800000 et 1900000 uniquement aux ÉtatsUnis (ce, malgré une politique restrictive adoptée depuis le début des années 80 par l'administration Reagan - Loescher et Scanlan, 1986 : 189) et un autre million se trouve réparti au Mexique, au Honduras, au Guatemala, au Costa Rica et à Belize. "(CCR, juin 1990 : 4). Au Canada, comme dans tous les autres pays développés, le nombre de réfugiés reste donc relativement minime en comparaison avec la masse de réfugiés dans les autres pays du monde. Ceux reconnus comme tels par le Canada le sont au sens de la Convention de Genève, c'est-à-dire en tant que " personnes qui, craignant avec raison d'être persécutées du fait de leur race, de leur religion, de leur nationalité, de leurs opinions politiques ou de leur appartenance à un groupe social, ne peuvent ou ne veulent retourner dans leur pays d'origine ou de résidence habituelle ». La deuxième catégorie d'admis sont « les membres de catégories désignées ", c'est-à-dire des personnes qui se trouvent dans une situation analogue à celle des réfugiés et qui ont besoin d'être rétablies, même si elles ne satisfont pas à la définition stricte de réfugié au sens de la Convention de Genève. Les trois catégories désignées sont les Indochinois, les prisonniers politiques et opprimés de l'Amé- 
rique latine et, jusqu'en 1991, les exilés volontaires des pays d'Europe de l'Est. Enfin, la troisième catégorie de réfugiés regroupe les gens qui bénéficient de mesures humanitaires spéciales soit parce qu'ils ont des parents au Canada (réunification des familles), soit parce qu'ils sont victimes d'une situation spéciale (guerre, séisme, inondation, etc.). II va sans dire que les ressortissants des pays touchés par ces mesures changent régulièrement chaque année.

Dans toutes ces catégories, de 15022 qu'il était en 1981, le nombre de réfugiés admis est passé à 28062 en 1988 (Commission de l'emploi et de l'immigration, 1989 : 47). En 1988, $43 \%$ (9 776) de ces réfugiés étaient parrainés par le secteur privé; $35 \%$ d'entre eux venaient d'Europe de I'Est, 25 \% (6 947) de l'Asie du Sud-Est (Viet-Nam, Laos, Cambodge) et $16 \%$ (4 605) de l'Asie occidentale. L'Amérique latine a fourni un contingent de 3959 réfugiés (13\%) et l'Afrique, 2119 (7\%).

En somme, les 39632 réfugiés à qui le Canada a permis l'entrée en 1990 ne forment qu'une infime proportion de nouveaux arrivants, en comparaison de ceux qui trouvent refuge dans les pays du tiers monde. Les plus pauvres portent le fardeau : 9 millions de réfugiés se retrouvent dans des pays de l'Asie du Sud-Est et 4500000 dans les pays d'Afrique.

\section{Les réfugiés face à un discours mystificateur}

Dans l'énoncé de politique en matière d'immigration et d'intégration, le discours au sujet des réfugiés fait montre d'une grande réserve et d'omissions assez évidentes (ministère des Communautés culturelles et de l'Immigration du Québec ( $\mathrm{MCCl}), 1991$ : 14). En premier lieu, on réduit l'interdépendance et la mondialisation à des " phénomènes économiques, écologiques et sociaux » et on passe sous silence le fait que la mondialisation est aussi politique, et que ce sont souvent des conflits militaro-politiques qui forcent des gens à quitter leur pays et à demander asile au Canada. Deuxièmement, on ramène les difficultés d'intégration au niveau des relations " entre personnes ayant des valeurs et des croyances différentes" (MCCl, $1990: 14)$. Enfin, le $\mathrm{MCCl}$ se situe dans une perspective d'accueil générale à l'égard des réfugiés. L'énoncé de politique souligne le caractère non discriminatoire de la politique mais, du même souffle, précise le cadre général de la sélection des réfugiés, soit la capacité d'accueil, ce qui en soi devient un critère d'exclusion fondamental :

L'attachement du Québec aux valeurs d'ouverture sur le monde et à la solidarité internationale se manifeste dans plusieurs orientations de la présente politique, notamment dans le caractère non discriminatoire de la sélection québécoise et dans l'engagement du Québec à 
accueillir, dans les limites de ses capacités, les personnes en situation de détresse. (MCCl, $1990: 14)$

Par ailleurs, force est de reconnaître que les principes de base de la politique québécoise s'inspirent d'un idéal indiscutable, la " tradition humanitaire ", pour accueillir des "personnes en situation de détresse » $(\mathrm{MCCl}, 1990: 24)$, mais les objectifs qui traduisent ces principes dans la pratique mettent une sourdine importante aux principes : que signifie "maintenir et mieux cibler l'accueil des réfugiés "? ( $\mathrm{MCCl}, 1990: 25)$. Ce libellé laconique soulève des questions que les porte-parole du gouvernement laissent toujours sans réponse claire : "Maintenir » signifie-t-il qu'on ne veut pas en augmenter le nombre? «Cibler l'accueil » implique-t-il restreindre l'accueil de certains groupes ? Si on y regarde d'un peu plus près, le gouvernement du Québec décore sa cape "humanitaire " de quelques fleurons, notamment l'accueil généreux et ouvert aux réfugiés indochinois au début des années $80(\mathrm{MCCl}, 1990$ : 37).

Tous les sens de l'énoncé de politique au sujet des réfugiés conduisent à un même carrefour : la correspondance aux intérêts économiques du Québec; le discours ramène tout à la capacité d'intégration liée aux attitudes des immigrants, des réfugiés et à celles de Québécois francophones à cet égard. En fond de scène, ce sont les intérêts économiques qui priment, même si l'énoncé de politique ne le dit pas toujours de façon claire.

Dans le cas des réfugiés :

le Québec détermine annuellement son objectif quantitatif ainsi que la répartition territoriale des personnes qu'il entend accueillir. Cette répartition s'appuie sur l'analyse de la situation mondiale et des caractéristiques des pays d'origine susceptibles de correspondre aux objectifs de la politique québécoise d'immigration. Dans tous les cas, la sélection individuelle tient compte de la capacité d'adaptation au Québec et de l'assistance offerte par des résidents québécois dans le cadre de divers programmes de parrainage. (MCCl, $1990: 37$ )

Un tel énoncé, par son amplitude et sa généralité, laisse place à une sélection qui frise l'arbitraire. Le Québec a, depuis quelques années, soutenu des projets dans les camps de réfugiés, notamment la tenue de cours de français, afin de faciliter l'établissement ultérieur au Québec. La politique d'immigration visant le redressement démographique, la prospérité économique, la pérennité du fait français et l'ouverture sur le monde, il est logique que l'admission des réfugiés s'inscrivent dans ces objectifs. Cependant, il faut y regarder de plus près.

La politique québécoise s'appuie d'abord sur les gens non seulement en mesure de subvenir à leurs besoins, mais aussi de s'adapter au Québec, de favoriser un accroissement de la consommation et 
notamment de créer des emplois, les investisseurs constituant $22 \%$ des nouveaux arrivants immigrés en 1989 ( $\mathrm{MCCl}, 1991$ : 31). Ce nouveau phénomène signifie un transfert de 208 millions de dollars en capitaux au Québec entre 1987 et 1990. Ce panorama reluisant peut sembler une politique adaptée; dans les faits, il s'agit d'une seule et même politique tout axée autour des immigrants les plus riches, les investisseurs, donc plus en mesure de s'adapter que les réfugiés. On en arrive à une situation où les réfugiés " sélectionnés " correspondent à peu près au profil de l'immigrant volontaire. Le paysan salvadorien, dirigeant syndical et politique, victime de la répression politico-militaire de régimes corrompus, tout comme les réfugiés fuyant l'Éthiopie, la Somalie, le Guatemala, le Koweit, etc., a bien peu de chance de traverser nos frontières s'il attend d'être sélectionné dans un camp.

Comme dans tous les pays développés, la politique d'immigration soumet les réfugiés à des procédures différentes qui les favorisent peu par rapport aux immigrants. Le bât blesse plus sérieusement et la contradiction s'accentue davantage à l'égard des revendicateurs du statut de réfugié : le revendicateur est presque présenté ouvertement comme un fraudeur, comme un " usurpateur », estimait l'ex-ministre fédérale de l'Immigration, Mme Barbara McDougall. Le gouvernement du Québec suit, mutatis mutandis, la même ligne de pensée. La lecture in extenso des éléments clés de l'énoncé de politique traduit bien cette contradiction dans le discours même.

Le Québec ne peut accepter que des personnes désireuses d'améliorer leur situation économique par l'immigration contournent la procédure régulière de sélection du Québec et du Canada, alors que d'autres candidats de ces mêmes pays acceptent de la respecter. En outre, la régularisation massive et fréquente du statut de personnes qui n'ont pas été sélectionnées nuit à l'atteinte des objectifs de la politique $d^{\prime}$ 'immigration. Elle rend notamment difficile la planification des volumes et de la composition du flux migratoire en fonction des priorités québécoises. De plus, la présence de quelque 35000 personnes en attente du statut de réfugié qui ont accès à divers services gouvernementaux - notamment dans les domaines de la santé et des services sociaux, de la sécurité du revenu et de l'éducation exerce une pression croissante sur les finances publiques. $\grave{A}$ cet égard, il faut rappeler que la capacité d'accueil des réfugiés n'étant pas illimitée, la reconnaissance du statut de réfugié à des personnes qui présentent une demande sur place réduit d'autant plus le nombre de personnes en situation de détresse sélectionnées à l'étranger, dans les camps où elles sont confinées. C'est pourquoi le Québec ne peut qu'approuver la volonté du gouvernement du Canada de limiter le mouvement des revendicateurs du statut de réfugié aux cas de détresse. (MCCl, $1990: 38-39)$ 
Une telle position conduit directement à une politique très restrictive et à une imitation servile de la stratégie fédérale, c'est-à-dire une dissuasion de la demande d'asile politique par des procédures bureaucratiques longues et lourdes. Le gouvernement québécois s'aligne sur les politiques et les stratégies fédérales, lesquelles, à ce jour, n'ont pas rendu justice aux revendicateurs. Cette politique de deux poids deux mesures contredit le discours humanitaire empreint d'un idéalisme naïf quant aux vertus de la sélection. Le gouvernement du Québec est tout à fait d'accord avec la politique du gouvernement du Canada qui doit mettre en œuvre des actions visant à « exercer un effet dissuasif auprès des personnes qui ne sont pas véritablement en situation de détresse " $(\mathrm{MCCl}, 1990: 39)$. Une telle stratégie, centrale au cœur de la politique fédérale et provinciale, vise avant tout à ajuster l'accueil des réfugiés aux " objectifs poursuivis lors du processus de règlement de l'arriéré des demandes de reconnaissance du statut de réfugié " et dans "la planification annuelle des intentions d'accueil de réfugiés, du nombre prévisible de revendicateurs dont le statut de réfugié est accordé au Québec » (MCCI, $1990: 39)$.

Tout comme la politique canadienne, la politique québécoise $s^{\prime}$ inscrit dans la visée du néo-libéralisme pratiqué dans les pays de l'Ouest par laquelle on prône la libre circulation des produits, des capitaux et de la main-d'œuvre, mais pas une "main-d'œuvre à risques "... Dans son ouvrage sur les politiques des pays occidentaux à l'égard des réfugiés, David Matas estime que non seulement les politiques tendent à empêcher l'entrée des réfugiés, mais elles sont discriminatoires à leur égard une fois qu'ils sont à l'intérieur des frontières. Certains pays vont même jusqu'à les empêcher de travailler et d'étudier. Il arrive à la conclusion que la majorité sont condamnés à une vie misérable dans les pays d'asile (Matas, $1989: 63-64$ ).

Au Canada, les mesures discriminatoires à l'égard des revendicateurs du statut de réfugié font régulièrement les manchettes. La plus célèbre est le fameux " arriérage " (mieux connu sous le nom de "backlog»). Le 25 octobre 1990, l'ex-ministre de I'Immigration, Mme Barbara McDougall, annonçait que 250000 immigrants seraient acceptés chaque année jusqu'en 1995, mais elle passait sous silence la situation des 100000 personnes toujours en attente du statut de réfugié au Canada. Depuis 1988, le ministère de l'Emploi et de l'Immigration n'a pas réussi à régler ces demandes. Au fur et à mesure que des décisions sont rendues, des milliers d'autres personnes entrent au pays et grossissent les rangs des revendicateurs.

Les procédures se révèlent trop bureaucratiques, mais elles servent d'abord des fins politiques. Le Conseil canadien pour les réfugiés estime qu'il s'agit là d'un constat d'échec, mais aussi d'une vaste opération politique pour convaincre l'opinion publique canadienne 
du danger de "I'invasion » du Canada par les réfugiés : « Force est de reconnaître, estime le CCR, que la perspective que le Canada soit envahi par des étrangers, et par surcroît des fraudeurs qui se précipiteraient à nos frontières dès l'annonce d'une mesure humanitaire, est bel et bien toujours présente dans les discours de la ministre." (CCR, novembre $1990: 2$ ). On laisse s'implanter l'idée que les revendicateurs ressemblent à des illégaux, estime aussi le CCR : "La présomption de fraude et d'illégalité sous-entendue dans les discours de la ministre reflète une attitude négative envers les revendicateurs. " (CCR, novembre $1990: 3$ ). Dans la même logique, les porte-parole

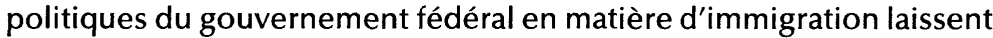
croire constamment que les investisseurs sont les seuls à favoriser le développement du Canada. Comme le souligne Filson (1983), la réciproque semble devenir une vérité éternelle: les réfugiés et les ouvriers immigrants constituent une menace pour l'emploi des autres travailleurs autochtones, ce qui suscite méfiance et xénophobie.

Dans ce contexte, on note aussi d'autres mesures nouvelles de plus en plus restrictives; le 16 avril 1990, par exemple, la ministre de I'Immigration d'alors décida de mettre fin au programme des prêts pour frais de transport des réfugiés parrainés à titre individuel. Avant cette mesure, les réfugiés sélectionnés à l'étranger avaient le choix entre deux formules pour venir s'établir au Canada : le parrainage du gouvernement ou le parrainage privé. Le parrainage privé amène les individus et les organismes à se porter garants du réfugié, car il n'a pas droit à l'aide sociale tout au long de sa première année de résidence. Pour aider le secteur privé, le gouvernement fédéral prêtait une somme équivalant aux frais de transport et le réfugié ou l'organisme devait le rembourser après une année de résidence. L'abolition de cette mesure a entraîné des résultats immédiats et directs : de nombreux parrainages furent refusés, surtout pour les plus démunis, les femmes et les jeunes. À la suite de cette décision, la politique de sélection des réfugiés et des immigrants a été fortement critiquée, " parce qu'elle favorise les plus nantis plutôt que les plus démunis et les plus en danger» (CCR, juin $1991: 9$ ).

Depuis quelques années, on observe aussi des mesures spéciales de harcèlement. En vertu du " danger pour la sécurité nationale », les services de renseignements canadiens se permettent $d^{\prime}$ interroger et de harceler des réfugiés. Ces pratiques contrastent étrangement avec les discours ronflants du premier ministre Mulroney : "La Confédération de demain, le Canada des années 90 est une terre promise, un peuple d'avenir, une société tolérante, un pays prospère et heureux parmi les nations du globe " (Secrétariat d'État, 1989 : 19).

En somme, le discours politique ne traduit pas la situation réelle et particulière des réfugiés et des revendicateurs du statut de réfugié. 
Pour eux, le " paradis ", c'est d'abord la difficulté de se faire admettre et, par la suite, de s'intégrer à leur nouvelle société. En principe, tant au Canada que dans les autres pays développés de l'hémisphère Nord, l'intégration renvoie à un processus de participation pleine et entière à la société (MCCl, 1990 : 19; $\mathrm{HCl}, 1991$ : 18; Schnapper, 1991 : 99). Cependant, il faut poser une question fondamentale : les réfugiés peuvent-ils participer de façon satisfaisante à la société ? De façon générale, on peut dire que s'ils s'intègrent, ils doivent redoubler d'efforts, car leur situation sociopolitique, leur visibilité et la perception souvent négative à laquelle ils se heurtent au travail, dans les quartiers et dans les services bloquent le processus d'intégration suivi par la masse des immigrants.

\section{Des services de deuxième classe}

C'est bien connu, les réfugiés constituent la majorité des utilisateurs des services sociaux spécialisés dans les questions interethniques, même si la plupart des services ne tiennent pas toujours compte de leurs caractéristiques et de leur situation particulière. La plupart des services relèvent des organismes non gouvernementaux privés. Devant cette situation, à partir de 1985, les deux niveaux de gouvernement ont diminué leur contribution financière à l'adaptation de façon substantielle, alors que le nombre de réfugiés utilisateurs des ONG a augmenté de façon considérable. Par exemple, en 1982-1983, le ministère des Communautés culturelles et de I'Immigration attribuait 3284659 \$, soit 13 \% de son budget global, à l'adaptation et à l'accueil, alors qu'en 1985 le nouveau gouvernement récemment élu de Robert Bourassa réduisait ce budget à $2568500 \$$, soit $9 \%$ du budget. En 1988, ce budget était encore réduit à $7 \%$ du budget global du $\mathrm{MCCl}$ et se chiffrait à 2438300 \$. C'est donc dire que de 1985 à 1988, le budget à l'adaptation et à l'accueil n'a pas augmenté, diminuant de 130 millions. De 1982 à 1988, il a diminué au total de 846 millions. En termes clairs, cela signifie qu'avec moins de ressources, les ONG doivent faire beaucoup plus.

Du côté fédéral, le Secrétariat d'État a aussi diminué son budget à I'adaptation. En 1985-1986, la portion du budget consacrée à ce qu'il nomme "le développement des groupes " était de 2508000 \$, soit $15 \%$ du budget global du Secrétariat d'État; en 1986-1987, le montant fut de $2525996 \$$, soit 14 \% du budget. Cela représente une augmentation de 17 millions seulement.

Parallèlement à ces coupures assez imposantes, à partir de la même époque, les ONG commencent à se plaindre de l'augmentation de "clientèle ". Le rapport annuel d'un organisme constate "qu'à 
partir de 1985, il y a une montée vertigineuse de la clientèle venant $d^{\prime}$ Afrique, d'Amérique latine et du Moyen-Orient. Cette population correspond à la catégorie " revendicateur au statut de réfugié... " (La Maisonnée, $1988: 2$ ). En somme, le nombre de demandes de services $s^{\prime}$ accroît et les ressources humaines et financières n'augmentent pas. Ce même constat s'applique aux dizaines d'ONG travaillant à l'accueil et à l'établissement des réfugiés.

En d'autres mots, les ONG sont soumis à des pressions incroyables pour assurer le financement de leurs services. Pour arriver à survivre, ils doivent faire preuve d'excellentes performances, c'est-à-dire prouver leur efficacité en offrant des services dans des conditions qui seraient jugées inacceptables par des professionnels et des employés du gouvernement : horaires difficiles, locaux inadéquats, emplois précaires, contrôles tatillons des fonctionnaires, etc.

Le désengagement de l'État est en marche depuis plusieurs années. Inutile de répéter ici tout le discours justificateur du passage au secteur privé, mais quelques paramètres suffisent à contextualiser ces nouvelles politiques. II ne s'agit pas seulement d'une rationalisation économique pour rendre les services rentables, c'est d'un nouveau mode de gestion du social qu'il s'agit. Efficacité et rentabilité étant les clés de voûte du discours des nouveaux idéologues de l'État chevillés à l'entreprise privée, il faut suivre les règles du marché en tout et susciter la compétition partout, même entre les organismes qui fournissent des services aux plus démunis, dont aux revendicateurs du statut de réfugiés. Pour en arriver là, l'État se déresponsabilise et s'en remet aux institutions locales qui, avec le retour en force de valeurs traditionnelles, $n$ 'ont pas trop de problèmes à compenser ce retrait de l'État par de multiples services reposant sur les épaules de généreux bénévoles à l'âme missionnaire.

Le discours des organismes sur leur rôle et leurs perspectives d'avenir révèle les difficultés rencontrées sur le plan organisationnel (financement, structure, gestion de la croissance, recrutement et formation du personnel, etc.) et sur le plan des « besoins » des usagers. Très souvent les usagers vivent des problèmes nés des contradictions dans les politiques gouvernementales sur le plan socio-juridique (par exemple, procédures d'enquête longues et complexes) et les ONG réduisent souvent ces difficultés au niveau strictement personnel pour être conformes aux exigences des bailleurs de fonds... Comme ces derniers ne sont pas intéressés à se faire contester, ils conditionnent les ONG à traiter d'abord les problèmes psychosociaux et non les problèmes socio-juridiques et socio-économiques.

La réponse des ONG s'en tient plutôt à une conception humaniste et philanthropique de leur pratique sociale. Le rapport annuel d'un organisme synthétise assez bien cette position assez généralisée : 
"Malgré tout, ces difficultés nous ont permis de découvrir autant chez les employés, les bénévoles et les membres du conseil d'administration de très belles qualités telles que le sens de l'organisation, un côté pratique remarquable, le sens du travail bien fait, de l'intelligence, du courage, du dévouement, de la tenacité, de la sincérité, etc. " (La Maisonnée, $1988: 2$ ). On met davantage l'accent sur les qualités morales des intervenants et des intervenantes, lesquels portent déjà une très lourde responsabilité, et on passe sous silence les causes structurelles des difficultés.

Est-il besoin d'ajouter à ces perspectives loin des visées pragmatiques de l'État que les organismes doivent se plier à une forme de soumission idéologique et politique. II ne faut pas créer de remous si I'on veut obtenir les prochaines subventions. L'expérience des ONG à ce chapitre est riche en leçons de toutes sortes, car plusieurs organismes ont goûté au couperet des bailleurs de fonds... En un sens, l'État définit le type d'ONG qu'il veut; il ne faut pas se surprendre de voir une panoplie d'ONG s'orienter surtout vers le dépannage sous diverses formes, puisque les possibilités de financement de la part de l'État sont de ce côté.

\section{Un partenariat à redéfinir}

Par ailleurs, l'État définit "ses » ONG comme des partenaires indispensables. De fait, en analysant les rapports annuels d'ONG, la plupart semblent intégrer cette notion de partenaires et reçoivent une forte proportion de "leur clientèle " des ministères et des services sociaux publics incapables d'accorder des services appropriés aux réfugiés.

Les partenaires non gouvernementaux se situent dans un rapport de force inégal à tous les points de vue : financement précaire et réduit, peu de pouvoir politique, peu de concertation, personnel expérimenté mais avec des conditions de travail difficiles (pas de sécurité d'emploi, peu de possibilité de se perfectionner, pas de régime de retraite, etc.). De plus, les ONG jouent un rôle palliatif important mais mal défini. On n'est pas encore passé de la suppléance ou de la complémentarité à un véritable partenariat. Les ONG ont une place extrêmement importante et nécessaire sur la scène sociale, mais le poids inégal entre eux, l'État et les services publics fait en sorte qu'après des années de pratique sociale valable, ils n'ont pas encore conquis l'espace politique qui leur permettrait de vivre un partenariat en toute justice. Quelques regroupements d'organismes comme " la table de concertation des organismes de Montréal pour les réfugiés" et l'Alliance des communautés culturelles pour l'égalité dans les ser- 
vices sociaux (ACCESS) ont réussi à ouvrir des brèches mais, dans les faits, un véritable partenariat dans les services sociaux entre l'État et les ONG reste encore à définir.

\section{Conclusion}

Le portrait paraît sombre. De fait, les réfugiés et les revendicateurs du statut de réfugié vivent une situation particulière et la question est posée : une politique d'accueil juste est-elle possible ? À long terme, il faut envisager un ordre mondial différent, une stratégie de développement axée sur une répartition plus juste des richesses et une recherche de la coopération au lieu de la domination politico-militaire et un contrôle des frontières qui devient de plus en plus difficile et inacceptable en raison de la gravité et de l'ampleur des problèmes conséquents à l'appauvrissement des pays du Sud. Entre l'idéal et la réalité, un immense gouffre reste à franchir dans le quotidien des pratiques sociales.

En premier lieu, s'imposent la promotion et la défense des droits des réfugiés et revendicateurs du statut de réfugié. Deuxièmement, la mobilisation doit tendre à faire changer les politiques restrictives, répressives et rétrogrades. Troisièmement, les divers paliers du réseau des services sociaux doivent développer des politiques et des stratégies d'intervention adaptées à la situation des réfugiés. Quatrièmement, le mouvement ouvrier et populaire d'ici doit associer les réfugiés à ses actions et à leurs revendications pour les droits socioéconomiques. Enfin, ONG et services sociaux publics doivent accorder une attention spéciale aux victimes de torture et aux jeunes réfugiés.

En travail social, on peut constater que la formation sur l'interven. tion en milieu pluriethnique s'enrichit peu à peu sur les plans théorique, idéologique et méthodologique. Le racisme, la xénophobie, l'analyse des situations sociales vécues par les réfugiés deviennent maintenant des éléments importants dans la formation universitaire des travailleurs sociaux et dans quelques programmes d'intervention $d^{\prime}$ institutions du réseau des services sociaux.

\section{Références bibliographiques}

ABOUD, Frances (1988). Children and Prejudice. Oxford: Basil Blackwell.

ANTHIAS, Floya (1990). "Race and class revisited - conceptualizing race and racism ", The Sociological Review, 38 (1) : 19-42.

BasRan, G.S. (1983). "Canadian Immigration Policy and Theories of Racism ", dans Li, Peter S. et Singh B. Bolaria (eds.), Racial Minorities in Multicultural Canada. Toronto : Garamond Press. 
BERRY, J.W. et R.C. ANNIS (eds.) (1987). Ethnic Psychology : Research and Practice with Immigrants, Refugees, Native Peoples, Ethnic Groups and Sojourners. Berwyn, PA : Selected Papers from a North American Regional Conference on the International Association for Cross-Cultural Psychology.

BolariA, Singh B. et Peter S. LI (1986). Racial Oppression in Canada. Toronto : Garamond Press.

BRETON, Raymond (octobre 1983). "La communauté ethnique, communauté politique ", Sociologie et Sociétés, XV (2) : 23-38.

CaSe, Charles E., Andrew M. Greeley et Stephan Fuchs (1989). "Social determinants of racial prejudice ", Sociological Perspectives, 32 (4) : 469-483.

CHAN, Kwok B. (1987). " Unemployment, Social Support and Coping : The Psychosocial Response of Indochinese Refugees to Economic Marginality ", dans Chan, Kwok B. et Doreen Marie Indra (eds.), Uprooting, Loss and Adaptation: The Resettlement of Indochinese Refugees in Canada. Canadian Public Health Association.

CHAN, Kwok B. et Doreen Marie INDRA (1987). Uprooting, Loss and Adaptation : The Resettlement of Indochinese Refugees in Canada. Canadian Public Health Association.

CHAN, Kwok B. et Lawrence LAM (1987). " Community Kinship and Family in the Chinese Vietnamese Community : Refugees Resettling in Quebec », dans Chan, Kwok B. et Doreen Marie Indra (editors), Uprooting, Loss and Adaptation: The Resettlement of Indochinese Refugees in Canada. Canadian Public Health Association.

CLARK, Russell D. et Anne MASS (1990). "The effects of majority size on minority influence ", European Journal of Social Psychology, 20 : 99-117.

Conseil canadien pour les réfugiés (juin 1990). Réfugiés, le point, $\mathrm{n}^{\circ} 1$.

Conseil canadien pour les réfugiés (novembre 1990). Réfugiés, le point, $\mathrm{n}^{\circ} 2$.

DORAIS, Louis-Jacques (1987). "Language Use and Adaptation », dans Chan, Kwok B. et Doreen Marie Indra (eds.), Uprooting, Loss and Adaptation : The Resettlement of Indochinese Refugees in Canada. Canadian Public Health Association.

FILSON, Glen (1983). "Class and ethnic differences in Canadian attitudes to native people's rights and immigration ", Revue canadienne de sociologie et d'anthropologie, 20 (4) : 454-482.

JACOB, André (1991a). Le racisme au quotidien. Montréal : CIDICHA.

JACOB, André et Jocelyne BERTOT (1991b). Les réfugiés, deux études de cas : les Salvadoriens et les Iraniens. Montréal : Éditions du Méridien.

JACOB, André et Jocelyne BERTOT (1991c). Intervenir avec les immigrants et les réfugiés. Montréal : Éditions du Méridien.

JUTEAU-LeE, Danielle (1983). "La production de l'ethnicité ou la part de l'idée ", Sociologie et Sociétés. XV (2) : 39-54.

LOESCHER, Gil et John A. SCANLAN (1986). Calculated Kindness : Refugees and America's Half-Open Door: 1945 to the Present. London : Free Press.

MATAS, David (1989). Closing the Doors. Toronto : Summerhill Press.

Ministère des Communautés culturelles et de l'Immigration du Québec (1990). Au Québec : pour bâtir ensemble. Énoncé de politique en matière d'immigration et d'intégration. Québec : Gouvernement du Québec.

SAMUEL, John (1987). "Economic Adaptation of Indochinese Refugees in Canada ", dans Chan, Kwok B. et Doreen Marie Indra (editors), Uprooting, Loss and Adaptation : The Resettlement of Indochinese Refugees in Canada. Canadian Public Health Association. 
SCHNAPPER, Dominique (1991). La France de l'intégration. Paris : Gallimard, 367 p. SIMON, Pierre-Jean (octobre 1983). "Le sociologue et les minorités : connaissance et idéologie, Sociologie et sociétés. XV (2) : 9-22.

SIRROS, Christos (novembre 1987). Rapport du comité sur l'accessibilité des services de santé et des services sociaux du réseau aux communautés culturelles. Rapport présenté à madame Thérèse Lavoie-Roux, ministre de la Santé et des Services sociaux, Québec.

VIDELIER, Philippe (septembre 1991). "Vieux fantasmes sur ces 'étranges étrangers'. Alerte : immigrés ! ", Le Monde Diplomatique, 38 (450) : 15.

WEINER, Gerry, secrétaire d'État du Canada et ministre d'État au multiculturalisme et à la citoyenneté (1989). L'élimination de la discrimination raciale au Canada. Ottawa : Gouvernement du Canada. 